\title{
Meaningful characterisation of perturbative theoretical uncertainties
}

\author{
N. Houdeau ${ }^{* a}$ and M. Cacciari, ${ }^{a b}$ \\ ${ }^{a}$ LPTHE, UPMC Univ. Paris 6 and CNRS UMR 7589, Paris, France \\ ${ }^{b}$ Université Paris Diderot, Paris, France \\ E-mail: nicolas.houdeau@lpthe.jussieu.fr, \\ matteo.cacciarielpthe.jussieu.fr
}

\begin{abstract}
We consider the problem of assigning a meaningful degree of belief to uncertainty estimates of perturbative series. We analyse the assumptions which are implicit in the conventional estimates made using renormalisation scale variations. We then formulate a Bayesian model that, given equivalent initial hypotheses, allows one to characterise a perturbative theoretical uncertainty in a rigorous way in terms of a credibility interval for the remainder of the series. We compare its outcome to the conventional uncertainty estimates in the simple case of the calculation of QCD corrections to the $e^{+} e^{-} \rightarrow$ hadrons process. We find comparable results, but with important conceptual differences. This work represents a first step in the direction of a more comprehensive and rigorous handling of theoretical uncertainties in perturbative calculations used in high energy phenomenology.
\end{abstract}

XXIst International Europhysics Conference on High Energy Physics

21-27 July 2011

Grenoble, Rhône-Alpes France

\footnotetext{
* Speaker.
} 


\section{Introduction}

The Large Hadron Collider (LHC) has finally been fired up and the race to collect data and analyse them has now started. While it is everybody's hope that discoveries will announce themselves in the form of unambiguous signals, it is of course conceivable, and probably also unavoidable initially, that they may rather present themselves cloaked under some subtle data/theory discrepancy. If this is the case, a full control of the uncertainty of the theoretical predictions becomes naturally of paramount importance: when comparing an experimental measurement to a theoretical calculation, we must be able to say if they agree or not, and with what degree of confidence we are making such statement. This is impossible to achieve unless both the experiment and the theory are provided with a meaningful (and commonly accepted) degree of uncertainty.

One area where progress has arguably not been made is in understanding the meaning of the residual theoretical uncertainty given by unknown higher orders in perturbation theory. The purpose of this work is to propose a framework to evaluate it. To achieve this we construct a model that leads to a well defined measure of credibility for a perturbative theoretical uncertainty, so that the degree of belief of a given interval can be explicitly calculated. Please refer to the publication [1] for a more complete presentation of the model and for a list of references. A full study can be found in the dissertation [2].

Even though the present work is of general interest, we specialize to the context of Quantum Chromodynamics (QCD): many LHC processes and backgrounds pertain to the QCD realm and, due to the relatively large size of the QCD coupling $\alpha_{S}$ and therefore the slower perturbative convergence, the issue of theoretical accuracy is more pressing.

\section{Definitions}

Consider the perturbative calculation for the cross section of a process taking place at a hard scale $Q$ performed at a renormalisation scale $\mu$.

$$
\sigma(Q)=\sum_{n=0}^{\infty} c_{n}(Q, \mu) \alpha_{S}^{n}(\mu)
$$

A concrete example is for instance the production of hadrons in $e^{+} e^{-}$collisions, the series $\sigma=$ $\sigma_{Q C D}$ being defined in this case by:

$$
\frac{\sigma\left(e^{+} e^{-} \rightarrow \text { hadrons }, Q\right)}{\sigma\left(e^{+} e^{-} \rightarrow \mu^{+} \mu^{-}, Q\right)}=R_{E W}(Q)\left(1+\sigma_{Q C D}(Q)\right)
$$

When no dependence is given explicitly, the coefficients and the coupling will be considered to be evaluated at a renormalisation scale $\mu=Q$. We also denote by

$$
\sigma_{k} \equiv \sum_{n=0}^{k} c_{n} \alpha_{S}^{n} \quad \text { and } \quad \Delta_{k} \equiv \sum_{n=k+1}^{\infty} c_{n} \alpha_{S}^{n}
$$

the partial sum up to the last calculated perturbative order $k$ and the remainder. Estimating the remaining uncertainty corresponds to giving an estimation of the order of magnitude of $\Delta_{k}$. 


\section{Conventional theoretical uncertainty estimate}

When truncated to a finite order, a perturbative calculation retains a higher-order dependence on the scale $\mu$. This dependence is generally exploited to estimate the presumed value of $\Delta_{k}$. One typically quotes an uncertainty interval $\left[\sigma_{k}^{-}, \sigma_{k}^{+}\right]$around $\sigma_{k}$, e.g.

$$
\sigma_{k}^{ \pm}=\sigma_{k} \pm \frac{\delta_{k}}{2} \quad \text { where } \quad \delta_{k} \equiv\left|\sigma_{k}(Q, 2 Q)-\sigma_{k}(Q, Q / 2)\right|
$$

A priori there is no reason why the interval $\left[\sigma_{k}^{-}, \sigma_{k}^{+}\right]$should estimate the remainder. However, assuming all the coefficients in the series share the same magnitude and that $\alpha_{S}$ is reasonably small

$$
\delta_{k} \simeq\left|\frac{d \sigma_{k}}{d \ln \mu^{2}}\right|_{\mu=Q}\left[\ln (2 Q)^{2}-\ln (Q / 2)^{2}\right] \simeq 3 k \beta_{0} \alpha_{S}^{k+1}\left|c_{k}\right|
$$

so that $\left|\Delta_{k}\right| \simeq \alpha_{S}^{k+1}\left|c_{k+1}\right| \sim \delta_{k}$.

Experience with perturbative calculations in QCD has shown that theoretical uncertainty estimates like those of eq. (3.1) are quite successful in predicting the range in which a higher order result will fall. Unfortunately, even if the hypothesis $\left|c_{n+1}\right| \simeq\left|c_{n}\right|$ is really correct and $\delta_{k}$ correctly describes the remainder of the series, there is no way of deciding how reliably it may do so.

\section{Credibility-based theoretical uncertainty estimate}

We establish a conditional density $f\left(\Delta_{k} \mid c_{0}, \ldots, c_{k}\right)$ for the remainder $\Delta_{k}$ in eq. (2.3), given the knowledge of the coefficients of the series up to order $k$. To this end we build a generic measure, i.e. a density function $f\left(c_{0}, c_{1}, \ldots\right)$ over the space of a priori unknown coefficients $c_{0}, c_{1}, \ldots$ We assume that all the coefficients $c_{n}$ in a perturbative series share some upper bound $\bar{c}>0$ to their absolute values, specific to the physical process studied. More precisely we suppose that:

Residual uncertainty: If we happened to know beforehand the parameter $\bar{c}$, our residual density for the value of an unknown coefficient $c_{n}$ would be

$$
f\left(c_{n} \mid \bar{c}\right)=\frac{1}{2 \bar{c}}\left\{\begin{array}{ll}
1 & \text { if }\left|c_{n}\right| \leq \bar{c} \\
0 & \text { if }\left|c_{n}\right|>\bar{c}
\end{array} \equiv \frac{1}{2 \bar{c}} \chi_{\left|c_{n}\right| \leq \bar{c}}\right.
$$

Mutual independence: When $\bar{c}$ is known, the residual uncertainties on the values of different coefficients are totally independent

$$
f\left(\left\{c_{i}, i \in I\right\} \mid \bar{c}\right)=\prod_{i \in I} f\left(c_{i} \mid \bar{c}\right) .
$$

Hidden parameter: $\quad \bar{c}$ is a priori totally unknown. We "define" a density for $\ln \bar{c}$ as the limit of a uniform distribution between $|\ln \varepsilon|$ and $-|\ln \varepsilon|$ when a small parameter $\varepsilon$ tends to zero:

$$
f_{\mathcal{E}}(\ln \bar{c})=\frac{1}{2|\ln \varepsilon|} \chi_{|\ln \bar{c}| \leq|\ln \varepsilon|} \Leftrightarrow f_{\mathcal{\varepsilon}}(\bar{c})=\frac{1}{2|\ln \varepsilon|} \frac{1}{\bar{c}} \chi_{\varepsilon \leq \bar{c} \leq 1 / \varepsilon}
$$

We perform every calculations using this $\varepsilon$-dependent density $f_{\varepsilon}$ with $\varepsilon \neq 0$, and the final result will then be the limit $\varepsilon \rightarrow 0$. 


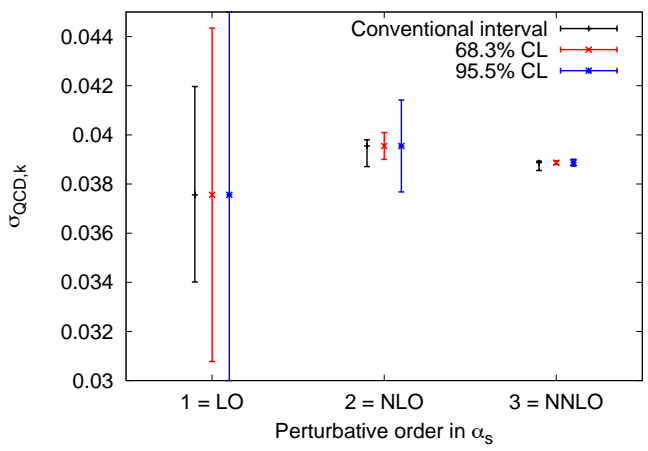

Figure 1: Uncertainty intervals for the $e^{+} e^{-} \rightarrow$ hadrons process, as given by the conventional method of scale variations (first interval on the left of each group) and by our model (the latter for $68.3 \%$ and $95.5 \%$ DoB, respectively middle and right of each group), for $\alpha_{S}=0.118$.

Using the three hypotheses eqs. (4.1), (4.2) and (4.3) one can show that

$$
f\left(c_{n} \mid c_{0}, \ldots, c_{k}\right)=\left(\frac{k+1}{k+2}\right) \frac{1}{2 \bar{c}_{(k)}}\left\{\begin{array}{cl}
1 & \text { if }\left|c_{n}\right| \leq \bar{c}_{(k)} \\
\frac{1}{\left(\left|c_{n}\right| / \bar{c}_{(k)}\right)^{k+2}} & \text { if }\left|c_{n}\right|>\bar{c}_{(k)}
\end{array} .\right.
$$

where $\bar{c}_{(k)} \equiv \max \left(\left|c_{0}\right|, \ldots,\left|c_{k}\right|\right)$. This parameter acts as an estimate of $\bar{c}$. Equating the remainder $\Delta_{k}$ to its first order $\alpha_{S}^{k+1} c_{k+1}$ and using eq. (4.4) we obtain:

$$
f\left(\Delta_{k} \mid c_{0}, \ldots, c_{k}\right) \simeq\left(\frac{k+1}{k+2}\right) \frac{1}{2 \alpha_{S}^{k+1} \bar{c}_{(k)}}\left\{\begin{array}{cl}
1 & \text { if }\left|\Delta_{k}\right| \leq \alpha_{S}^{k+1} \bar{c}_{(k)} \\
\frac{1}{\left(\left|\Delta_{k}\right| /\left(\alpha_{S}^{k+1} \bar{c}_{(k)}\right)\right)^{k+2}} & \text { if }\left|\Delta_{k}\right|>\alpha_{S}^{k+1} \bar{c}_{(k)}
\end{array} .\right.
$$

This result depends on the entire set of the calculated coefficients via the parameter $\bar{c}_{(k)}$.

It is now possible to assign a degree of belief (DoB) to the conventional intervals defined in (3.1), where $\delta_{k}$ is given by its approximation (3.2):

$$
\mathbb{C}\left(\Delta_{k} \in\left[-\frac{\delta_{k}}{2}, \frac{\delta_{k}}{2}\right] \mid c_{l}, \ldots, c_{k}\right)=\left\{\begin{array}{cl}
1-\frac{1}{n_{c}+1}\left[\frac{2}{3 k \beta_{0}} \frac{\bar{c}_{(k)}}{\left|c_{k}\right|}\right]^{n_{c}} & \text { if } \frac{\delta_{k}}{2} \geq \alpha_{S}^{k+1} \bar{c}_{(k)} \Leftrightarrow\left|c_{k}\right| \geq \frac{2}{3 k \beta_{0}} \bar{c}_{(k)} \\
\frac{n_{c}}{n_{c}+1} \frac{3 k \beta_{0}}{2} \frac{\left|c_{k}\right|}{\bar{c}_{(k)}} & \text { if } \frac{\delta_{k}}{2}<\alpha_{S}^{k+1} \bar{c}_{(k)} \Leftrightarrow\left|c_{k}\right|<\frac{2}{3 k \beta_{0}} \bar{c}_{(k)}
\end{array}\right.
$$

$n_{c}$ is the number of calculated coefficients of the series $\left(n_{c}=k+1\right.$ when the series starts at order $l=0$ as in the previous expressions). In the $e^{+} e^{-} \rightarrow$ hadrons case at $90 \mathrm{GeV}$, the DoBs of the different conventional intervals would be $46 \%$ at LO, $90 \%$ at NLO and $98.8 \%$ at NNLO.

Better still, the knowledge of $f\left(\Delta_{k} \mid c_{0}, \ldots, c_{k}\right)$ allows one to compute the smallest $p \%$-credible interval for $\Delta_{k}$. It turns out to be centred at zero, and hence we denote it by $\left[-d_{k}^{(p)}, d_{k}^{(p)}\right]$. Using the analytical approximation in eq. (4.5)

$$
d_{k}^{(p)}=\left\{\begin{array}{cl}
\alpha_{S}^{k+1} \bar{c}_{(k)} \frac{n_{c}+1}{n_{c}} p \% & \text { if } p \% \leq \frac{n_{c}}{n_{c}+1} \\
\alpha_{S}^{k+1} \bar{c}_{(k)}\left[\left(n_{c}+1\right)(1-p \%)\right]^{-1 / n_{c}} & \text { if } p \%>\frac{n_{c}}{n_{c}+1}
\end{array}\right.
$$

In the $e^{+} e^{-} \rightarrow$ hadrons case at $90 \mathrm{GeV}$, the $68.3 \%$ and $95.5 \%$ confidence level intervals are pictured on the figure 1 alongside conventional intervals. 


\section{Conclusion}

We have introduced a Bayesian model which allows one to characterise in terms of intervals of a given degree of belief (or degree of belief of a given interval) the residual theoretical uncertainty of a perturbative calculation. Our aim is to put on more solid ground the estimate of the uncertainty of a known result, not to improve in any way the calculation itself. This we try to achieve by formalising hypotheses on the behaviour of the coefficients of perturbative series, and then by deriving from these hypotheses the degree of belief values in a rigorous way.

We have chosen to try to translate as closely as possible into our model the assumption which is implicitly made when employing the conventional method (scale variations) for estimating the uncertainty, namely that successive coefficients of a perturbative series tend to have similar size. One may or may not believe this hypothesis to be well grounded, and our choice is not necessarily true or even just the best possible one. However, what matters, and what this paper wants to provide, is not so much which hypotheses are made, but rather the formalism that allows one to derive from them a proper characterisation of the residual theoretical uncertainty: our framework can then be considered as a box into which to input one's favourite hypothesis about the behaviour of a series, and from which to extract the appropriate degree of belief values.

\section{References}

[1] M. Cacciari and N. Houdeau, "Meaningful characterisation of perturbative theoretical uncertainties," JHEP 1109 (2011) 039 [arXiv:1105.5152 [hep-ph]].

[2] N. Houdeau "LHC phenomenology, theoretical predictions and their uncertainties in a bayesian framework," http://tel.archives-ouvertes.fr/tel-00640266 